\title{
Sphingosine 1-phosphate promotes endothelial cell barrier integrity by Edg-dependent cytoskeletal rearrangement
}

\author{
Joe G.N. Garcia, ${ }^{1}$ Feng Liu, ${ }^{1}$ Alexander D. Verin, ${ }^{1}$ Anna Birukova,,${ }^{1}$ Melissa A. Dechert, ${ }^{2}$ \\ William T. Gerthoffer, ${ }^{2}$ James R. Bamburg, ${ }^{3}$ and Denis English ${ }^{4}$ \\ ${ }^{1}$ Division of Pulmonary and Critical Care Medicine, Johns Hopkins University School of Medicine, Baltimore, Maryland, USA
2Department of Pharmacology, University of Nevada School of Medicine, Reno, Nevada, USA
${ }^{3}$ Department of Biochemistry, Colorado State University, Fort Collins, Colorado, USA
${ }^{4}$ Methodist Research Institute, Indianapolis, Indiana, USA
}

Address correspondence to: Joe G.N. Garcia, Johns Hopkins Asthma and Allergy Center, 5501 Hopkins Bayview Circle, 4B-77, Baltimore, Maryland 21224-6801, USA. Phone: (410) 550-5961; Fax: (410) 550-6985; E-mail: drgarcia@jhmi.edu.

Received for publication February 5, 2001, and accepted in revised form July 24, 2001.

\begin{abstract}
Substances released by platelets during blood clotting are essential participants in events that link hemostasis and angiogenesis and ensure adequate wound healing and tissue injury repair. We assessed the participation of sphingosine 1-phosphate (Sph-1-P), a biologically active phosphorylated lipid growth factor released from activated platelets, in the regulation of endothelial monolayer barrier integrity, which is key to both angiogenesis and vascular homeostasis. Sph-1-P produced rapid, sustained, and dose-dependent increases in transmonolayer electrical resistance (TER) across both human and bovine pulmonary artery and lung microvascular endothelial cells. This substance also reversed barrier dysfunction elicited by the edemagenic agent thrombin. Sph-1-P-mediated barrier enhancement was dependent upon $\mathrm{G}_{\mathrm{i} \alpha}$-receptor coupling to specific members of the endothelial differentiation gene (Edg) family of receptors (Edg-1 and Edg-3), Rho kinase and tyrosine kinase-dependent activation, and actin filament rearrangement. Sph-1-P-enhanced TER occurred in conjunction with Rac GTPase- and p21-associated kinase-dependent endothelial cortical actin assembly with recruitment of the actin filament regulatory protein, cofilin. Platelet-released Sph-1-P, linked to Rac- and Rho-dependent cytoskeletal rearrangement, may act late in angiogenesis to stabilize newly formed vessels, which often display abnormally increased vascular permeability.
\end{abstract}

J. Clin. Invest. 108:689-701 (2001). DOI:10.1172/JCI200112450.

\section{Introduction}

An increase in vascular permeability is a cardinal feature of inflammation and an essential component of tumor metastasis, angiogenesis, and atherosclerosis. Proteins and lipids released after platelet activation have long been appreciated as enhancing the integrity of the microcirculation in vivo and in vitro $(1,2)$. In contrast, a reduction in circulating platelets in humans accelerates capillary leakage and tissue edema formation, results verified in studies of platelet depletion in animals $(3,4)$. Although the exact mechanism underlying the effects of thrombocytopenia have not been clearly defined, this compromise in the endothelial barrier can be reversed either with platelet infusions or by the systemic administration of platelet-released products (5). Several platelet-derived lipids have emerged as important signaling molecules that exert their effects by inducing endothelial cell activation (6). For example, lysophosphatidic acid (LPA), a lipid released from thrombin-activated platelets, has stabilizing effects on vascular integrity $(7,8)$. However, recent studies suggest that only small amounts of LPA are generated in stimulated platelets (9), and LPA has also been implicated in increased neuronal endothelial monolayer leakiness (10), an effect that calls into question its proposed role in decreasing vascular permeability. Thus, the physiological role of LPA as an important regulator of vascular barrier permeability remains controversial.

There has been considerable interest in diverse endothelial cell responses, including angiogenesis, to other platelet-derived phospholipids, including phosphatidic acid (PA) (11) and more recently sphingosine 1-phosphate (Sph-1-P) $(6,8,12-16)$, which, unlike LPA, is generated and released in abundant quantities by platelets (9). Angiogenesis is a multifaceted process that begins with increases in vascular permeability in order to liberate endothelial cells from existing vessels and terminates with stabilization of permeability characteristics of newly formed vessels. The initial step, that is, increased permeability, is thought to be driven by liberation of endothelial cells from established monolayers, a process that may involve metalloproteinases (17). Endothelial cells then migrate chemotactically to their final destination, proliferate in response to angiogenic factors, and undergo capillary morphogenesis. Angiogenic differentiation, a process that may be induced by the same factor or by different factors that drive chemotactic migration, results in morphogenic alterations and the formation of new capillaries, which are rather leaky and therefore not fully functional $(18,19)$. 
We have hypothesized (8) that other factors, including those released by stimulated platelets, impinge upon these newly formed vessels to enhance their integrity and thereby impart functionality, the final feature of the angiogenic response.

Sph-1-P has been demonstrated to be a remarkably effective endothelial cell agonist that induces proliferation, calcium mobilization, adhesion molecule expression, and suppression of apoptosis $(6,14,20-22)$. Present in nanomolar to micromolar concentrations in human and animal serum (22), Sph-1-P is avidly released by stimulated platelets and binds to $G$ protein-coupled receptors encoded by members of the endothelial differentiation gene (Edg) family of receptors (14-16). Edg-1 was cloned from RNA expressed in endothelial cells stimulated to undergo angiogenic responses in vitro (23) and, together with Edg-3, -5 , and -6 , appears to be a high-affinity Sph-1-P receptor (24-26). In contrast, LPA preferentially binds Edg-2, -4, -7 , and -8 , and perhaps other unidentified members of this family of receptors $(6,25,26)$. Recent reports, including our own, have unequivocally demonstrated that Sph-1-P evokes diverse cell-specific responses, including endothelial cell mitogenesis and chemotaxis $(8,12-15,26,27)$. We have found that Sph-1-P released from platelets during clotting is the major source of the endothelial cell chemoattractant activity of serum, and after migration induces endothelial cells to proliferate avidly and differentiate forming capillary-like multicellular structures indicative of early blood vessel formation (12). In vivo, Sph-1-P was strikingly effective in avian chorioallantoic membranes (6), in Matrigelimplanted plugs in mice (14), and in the avascular mouse cornea (12). These studies demonstrate that blood coagulation initiates angiogenic responses through the release of Sph-1-P, which exerts optimal effects in concert with protein growth factors.

Despite this rapid increase in our understanding of Sph-1-P involvement in several defined steps in the angiogenic process, the effect of Sph-1-P on vascular barrier function has not been investigated. Therefore, we examined the effect of Sph-1-P on endothelial cell permeability and demonstrate that at physiologically relevant concentrations Sph-1-P exhibits potent barrier protection via Edg receptor ligation and $\mathrm{G}_{\mathrm{i} \alpha}$-linked Rhoand Rac GTPase-dependent cytoskeletal alterations. Sph-1-P may be involved in a critical linkage between coagulation, inflammation, and angiogenesis working in concert with other factors to initiate neovascularization and to potentiate the function of newly formed vessels by optimizing endothelial barrier integrity.

\section{Methods}

Reagents. Chemicals, including synthetic Sph-1-P, were obtained from Sigma Chemical Co. (St. Louis, Missouri, USA), unless noted otherwise. Metabolic inhibitors including genistein, LY-294002, ML-7, SB-203580, PP2, UO126 and 1,2-bis (aminophenoxy) ethane- $\mathrm{N}, \mathrm{N}, \mathrm{N}^{\prime}, \mathrm{N}^{\prime}$-tetraacetic acid tetrakis (acetoxy- methylester) (BAPTA-AM) were from Calbiochem-Novabiochem Corp. (La Jolla, California, USA) and were dissolved in DMSO at a concentration of 1-10 mM. Pertussis toxin (Calbiochem-Novabiochem Corp.) was dissolved in water at $500 \mu \mathrm{g} / \mathrm{ml}$. FBS was acquired from HyClone Laboratories (Logan, Utah, USA). Tissue culture media and growth supplements were obtained from Life Technologies Inc. (Grand Island, New York, USA). The Rho kinase inhibitor, Y27632, and Rac activity assay kit were obtained from Upstate Biotechnology Inc. (Lake Placid, New York, USA). FuGENE6 transfection reagent was purchased from Boehringer Mannheim Biochemicals Inc. (Indianapolis, Indiana, USA). Lipofectamine and Opti-MEM were from Life Technologies Inc. (Rockville, Maryland, USA). All the reagents used for immunofluorescence studies were from Molecular Probes Inc. (Eugene, Oregon, USA). Polyclonal rabbit anti-HA tag $\mathrm{Ab}$, monoclonal mouse anti-c-myc tag $\mathrm{Ab}$, and anti-p21-associated kinase (anti-PAK-1) Ab were purchased from Santa Cruz Biotechnology Inc. (Santa Cruz, California, USA). Specific anti-monophosphorylated myosin light chain (MLC) antisera were kindly provided by Michael $\mathrm{T}$. Crow (NIH, Baltimore, Maryland, USA). Dominant negative myc-tagged PAK-1 construct and constitutively active HA-tagged Rac construct (Rac V12) were kindly provided by M. Nicolic (Harvard Medical School, Boston, Massachusetts, USA). Anti-myosin IIa Ab was purchased from BAbCO (Richmond, California, USA), and anti-cofilin $\mathrm{Ab}$ was purchased from Cytoskeleton Inc. (Denver, Colorado, USA).

Human and bovine pulmonary artery endothelial cell cultures. Bovine pulmonary artery endothelial cells were obtained from American Type Culture Collection (Manassas, Virginia, USA) and bovine microvascular endothelium from Cell Systems (Kirkland, Washington, USA). Both cell types were cultured in complete DMEM supplemented with $20 \%$ (vol/vol) FCS, $15 \mu \mathrm{g} / \mathrm{ml}$ endothelial cell growth supplement, $1 \%$ antibiotic and antimycotic solution (penicillin, 10,000 $\mathrm{U} / \mathrm{ml}$; streptomycin, $10 \mu \mathrm{g} / \mathrm{ml}$; and amphotericin $\mathrm{B}$, $25 \mu \mathrm{g} / \mathrm{ml}$ ), and $0.1 \mathrm{mM}$ nonessential amino acids, as previously described $(27,28)$. Human pulmonary artery endothelial cells were obtained from Clonetics Corp. (Walkersville, Maryland, USA) and cultured in EBM-2 complete medium (Clonetics Corp.). Endothelial cell cultures were maintained at $37^{\circ} \mathrm{C}$ in a humidified atmosphere of $5 \% \mathrm{CO}_{2}-95 \%$ air and grown to contactinhibited monolayers with typical cobblestone morphology. Cells from each primary flask were detached with $0.05 \%$ trypsin, resuspended in fresh culture medium, and passaged into eight-well ECIS electrode arrays for electrical resistance determinations, $100-\mathrm{mm}^{2}$ dishes for transfection or 12-well plate (with cover glasses) for immunofluorescent analysis.

Measurement of endothelial cell electrical resistance. Endothelial cells were grown to confluence in polycarbonate wells containing evaporated gold microelectrodes (surface area, $10^{-3} \mathrm{~cm}^{2}$ ) in series with a large 
gold counter electrode $\left(1 \mathrm{~cm}^{2}\right)$ connected to a phasesensitive lock-in amplifier as described previously (29, 30,31). Measurements of transendothelial electrical resistance (TER) were performed using an electrical cell-substrate impedance sensing system (ECIS) (Applied BioPhysics Inc., Troy, New York, USA). Briefly, current was applied across the electrodes by a $4,000-\mathrm{Hz}$ AC voltage source with amplitude of $1 \mathrm{~V}$ in series with a $1 \mathrm{M} \Omega$ resistance to approximate a constant current source $(\sim 1 \mu \mathrm{A})$. The in-phase and out-ofphase voltages between the electrodes were monitored in real time with the lock-in amplifier and subsequently converted to scalar measurements of transendothelial impedance, of which resistance was the primary focus. TER was monitored for 30 minutes to establish a baseline resistance $\left(R_{0}\right)$ which, for bovine pulmonary endothelium, was typically between 8 to $12 \times 10^{3} \Omega$ (wells with $R_{0}<7 \times 10^{3} \Omega$ were rejected). As cells adhere and spread out on the microelectrode, TER increases (maximal at confluence), whereas cell retraction, rounding, or loss of adhesion is reflected by a decrease in TER (32). These measurements provide a highly sensitive biophysical assay that indicates the state of cell shape and focal adhesion (29). Values from each microelectrode were pooled at discrete time points and plotted versus time as the mean $\pm \mathrm{SE}$ of the mean.

Treatment of endothelial cells with Edg-1 and Edg-3 antisense oligonucleotides. Phosphothioate oligonucleotides specific for Edg-1 and Edg-3, as well as control oligonucleotides (sense or scrambled sequence), were synthesized by the DNA Analysis Facility at Johns Hopkins University (Baltimore, Maryland, USA) based upon the published human Edg-1 and Edg-3 sequences (23) and their specificity determined previously by our laboratory (27). Bovine pulmonary artery endothelial cells grown to $80-90 \%$ confluence in ECIS plates were treated for 4 hours with $10 \mu \mathrm{g} / \mathrm{ml}$ (approximately $1.8 \mu \mathrm{M}$ ) of oligonucleotides complexed with $10 \mu \mathrm{g} / \mathrm{ml}$ of Lipofectamine in Opti-MEM as we described previously (27). An equal volume of complete endothelial growth media containing $10 \%$ FBS was then added directly to the wells, and the cells were incubated for 20 hours. The medium was then replaced by M199 containing $10 \mu \mathrm{g} / \mathrm{ml}$ oligonucleotides, and the cells were incubated for 24 hours followed by incubation with complete culture medium containing $10 \mu \mathrm{g} / \mathrm{ml}$ oligonucleotides for additional 24 hours before TER measurements. In parallel studies, cell lysates were harvested after Edg-1 and scrambled oligonucleotide challenge and subjected to Western blot analysis using Edg-1 antisera (27).

Cotransfection of specific plasmids with enhanced green fluorescent protein. To obtain high transfection efficiencies for functional analysis, cells were cotransfected with plasmids expressing the selectable green fluorescent protein (GFP) and the plasmid of interest. Briefly, endothelial cells grown to $50-60 \%$ confluence in $100-\mathrm{mm}$ dishes were transiently transfected with enhanced green fluorescent protein ( $\mathrm{pEGFP}$ ) in combination with one of a variety of plasmids, including those encoding the $G$ protein $\beta \gamma$-binding domain of $\beta A R K 1$ (27), the $\mathrm{G}_{\mathrm{i} \alpha 2}$ inhibitory minigene $(13,27)$, and Edg- $1,-3$, and -5 receptors. The BARK minigene plasmid (pRK- $\beta$ ARK1-495-689) contains the carboxyl terminus of the $\beta$-adrenergic receptor kinase (the $G \beta \gamma$-binding domain) and was kindly provided by Walter J. Koch (Duke University, Durham, North Carolina, USA). The $\mathrm{G}_{\mathrm{i} \alpha 1 / 2}$ minigene encodes a peptide corresponding to the last 11 residues of $\mathrm{G}_{\mathrm{i \alpha 1} 1 / 2}$ carboxyl terminal (344-354), and the peptide inhibits specific receptor- $G_{i}$ protein interactions (33). Edg receptor plasmids were generously provided by Nicholas Aniellin (University of Connecticut, Storrs, Connecticut, USA) and encode complete Edg receptor sequences (14). Cells were incubated with DNA/FuGENE6 mixture prepared as follows: $50 \mu \mathrm{l}$ FuGENE6 was diluted in $1 \mathrm{ml}$ Opti-MEM, incubated at room temperature for $15 \mathrm{~min}$ utes, and added to $5 \mu \mathrm{g}$ DNA containing $1 \mu \mathrm{g}$ pEGFP and $4 \mu \mathrm{g}$ of either $\beta A R K 1 ; \mathrm{G}_{\mathrm{i} \alpha 2} ; \mathrm{Edg}-1,-3$, or -5 ; or their respective vector controls. The mixtures were then incubated at room temperature for 30 minutes and added to dishes containing $10 \mathrm{ml}$ fresh complete medium. After 24-hour incubation, cells were trypsinized, centrifuged, and resuspended in complete medium. Cells expressing GFP were isolated by FACS using a FACStarplus cell sorter (Becton Dickinson Immunocytometry Systems, San Jose, California, USA). As described previously, approximately $60-90 \%$ of cells selected in this manner carry the cotransfected plasmid of interest along with the selectable GFP plasmid (13), whereas transfection efficiencies were considerably lower than $20 \%$ in the absence of cell sorting. The selected GFP-positive cells were placed in ECIS wells and cultured for 2 days followed by TER.

Immunofluorescence microscopy. Endothelial cells grown on gelatinized cover slips were incubated with Sph-1-P or vehicle control, fixed in 4\% paraformaldehyde for 10 minutes, washed three times with PBS, permeabilized with $0.2 \%$ Triton X-100 in PBS for 5 minutes, and blocked with $2 \%$ BSA in PBS for 30 minutes. Incubations with primary Ab's of interest (myosin heavy chains, PAK-1, cofilin, phosphorylated MLC) were performed in blocking solution (2\% BSA in PBS) for 1 hour at room temperature. After three washes with PBS, cells were incubated with appropriate secondary Ab's conjugated to immunofluorescent dyes (Alexa 488 for green fluorescence or Alexa 546 for red fluorescence) in blocking solution for 1 hour at room temperature. Actin filaments were visualized by staining cells with Texas red-conjugated phalloidin for 1 hour at room temperature. After three washes with PBS, the cover slips were mounted using Slow Fade kit (Molecular Probes Inc.). Analyses of immunofluorescent staining were performed using a Nikon Eclipse TE 300 microscope with $\times 60$ objective lens and Sony Digital Photo camera DKC 5000.

Determination of Rac GTPase activity. Endothelial cells grown in 100-mm dishes were incubated with agonist for indicated time periods in serum-free M199. Cells were lysed in $500 \mu$ lysis buffer and homogenized by pipetting. 


\begin{abstract}
Figure 1
Sph-1-P enhances endothelial cell barrier properties. Endothelial cells were plated on gold microelectrodes to measure TER and were cultured to confluence. Growth medium was replaced with serum-free M199, and after equilibration and stabilization, cells were challenged with agonist (Sph-1-P or thrombin). Data are representative of multiple independent experiments (minimum of three). Shown are the responses to increasing concentrations of Sph-1-P in bovine pulmonary artery endothelial cells (BPAEC) (a) and in bovine lung microvascular endothelial cells (BLMVEC) (b). (c) The effect of Sph-1-P on thrombin-induced barrier dysfunction in human pulmonary artery endothelial cells (HPAEC). In these experiments, endothelial cells were simultaneously treated with Sph-1-P ( $1 \mu \mathrm{M}, 30$ minutes) and with the potent edemagenic agent thrombin (100 nM) (38). Sph-1-P consistently elicits a significant increase in TER, which returns to baseline control values in the presence of thrombin but does not exhibit the $40 \%$ decline below control values produced by thrombin alone. Furthermore, Sph-1-P rapidly and consistently restores human endothelial cell barrier integrity in cells stimulated previously with thrombin (d).
\end{abstract}

a
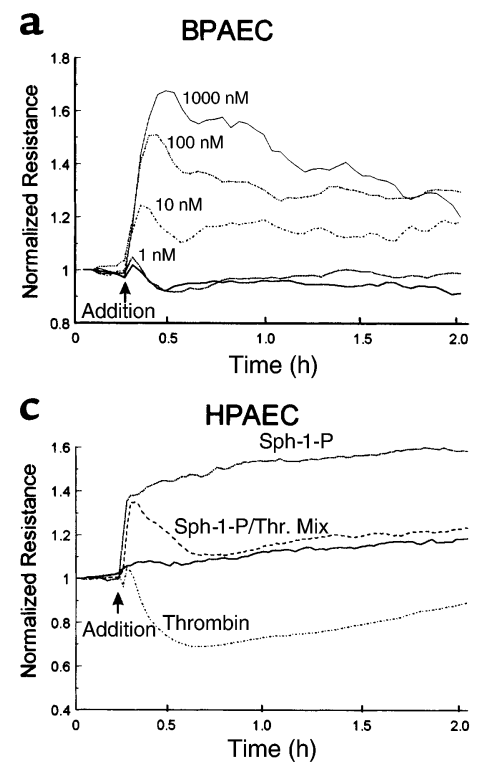

b
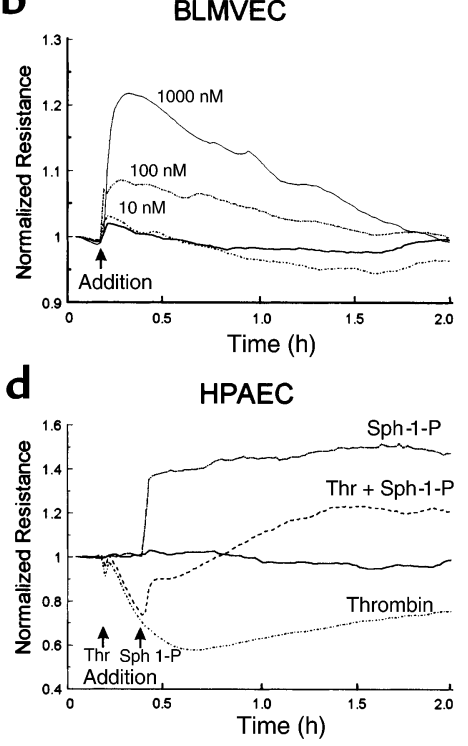

After a brief centrifugation to remove the cell debris, 300 $\mu \mathrm{l}$ of supernatants were incubated with human $\mathrm{p} 21$-binding PAK-1 domain (residues 67-150), conjugated with agarose $(10 \mu \mathrm{g}, 30$ minutes; Upstate Biotechnology Inc.). The agarose beads were washed with the lysis buffer (five times) and resuspended in $30 \mu \mathrm{l}$ of $2 \times$ SDS gel loading buffer. The samples were subjected to electrophoresis in $15 \%$ gel, and protein was detected using anti-Rac mAb. For total Rac detection, $5 \mu \mathrm{l}$ of the original cell lysates was subjected to electrophoresis.

\section{Results}

Sph-1-P enhances endothelial cell barrier properties. Sph-1-P strikingly and consistently enhanced transmonolayer electrical resistance (TER) across human and bovine endothelial cell monolayers in a dose-dependent manner consistent with barrier protection (Figure 1). Maximal protection was obtained with $1 \mu \mathrm{M}$ of Sph-1-P with TER measurements that peaked after approximately 10-20 minutes and remained above baseline values for several hours (Figure 1, a and b). Substantially higher concentrations of Sph-1-P (up to $50 \mu \mathrm{M}$ ) did not further increase TER but were variably followed by significant declines in barrier integrity after 30 minutes (data not shown). Sph-1-P evoked comparable TER responses in both bovine and human pulmonary artery endothelium (Figures 1, a, c, and d) as well as in bovine microvascular lung endothelial cells (Figure 1b). In addition to the direct effect of Sph-1-P on human endothelial cell TER values, simultaneous treatment with Sph-1-P and the potent edemagenic agent, thrombin, significantly attenuated the approximately $40 \%$ decline in TER typically observed after thrombin stimulation (Figure 1c). Furthermore, Sph-1-P fully restored vascular barrier integrity when added subsequent to thrombin challenge (Figure 1d). Thus, the phospholipid growth factor Sph-1-P not only increases the barrier integrity of untreated human and bovine endothelial monolayers, but also effectively protects endothelial cell monolayers from the barrier disruptive effects of edemagenic agents such as thrombin.

Sph-1-P-mediated endothelial cell barrier protection involves Edg receptor ligation and $G_{i \alpha}$ protein coupling. We undertook experiments designed to define the basic elements of the cellular signaling pathways by which Sph-1-P exerts its barrier-protective effects on the vascular endothelium. Indeed, Sph-1-P may enter cells through their plasma membranes where it may act intracellularly by activating cytosolic enzymes or membrane-bound constituents. Alternatively, extracellular Sph-1-P may exert effects via receptor-dependent as well as by receptor-independent mechanisms $(26,34,35)$. Figure 2 provides several lines of evidence that implicate Edg receptor ligation in the initiation of Sph-1-P-dependent signal transduction pathways that lead to endothelial barrier enhancement. Our previous studies of Edg receptor expression in human pulmonary artery endothelial cells using RT-PCR demonstrated the abundant expression of Edg-1, very modest expression of Edg-3, and no expression of Edg-5 (27). Specific downregulation of either Edg-1 (Figure 2a) or Edg-3 receptor expression by antisense oligonucleotides, which we have shown to inhibit Sph-1-P-induced endothelial cell chemotaxis (27), significantly attenuated Sph-1-P-induced increases in TER (Figure 2b). We next examined endothelial cells transfected with plasmids expressing Edg-1, Edg-3, or Edg-5 receptors and found that whereas neither Edg-1 nor Edg-3 overexpression altered TER responses to Sph-1-P, Edg-5 overexpression significantly enhanced Sph-1-P-stimulated TER responses in bovine endothelium (Figure 2c). These results suggest that sufficient Edg- 1 and -3 receptors exist to assure Sph-1-P enhancement of endothelial cell barrier integrity by igniting a signaling cascade after primary ligation to Edg-1 and possibly Edg-3. Ligation of Edg-5, although not normally expressed, also appears to be linked to barrier-protective signaling pathways. 
We, and others, have reported that Sph-1-P increases endothelial cell chemotaxis and other angiogenic responses in a pertussis toxin-sensitive (PTX-sensitive) fashion, consistent with Edg- $\mathrm{G}_{\mathrm{i} \alpha}$ protein-coupled receptor signaling $(12,26,27,35,36)$. Similar PTX treatment ( 2 hours, $200 \mathrm{ng} / \mathrm{mL}$ ) abolishes $\mathrm{G}_{\mathrm{i} \alpha}$-mediated signaling via complete ADP ribosylation of PTX G protein substrates $(27,28)$ (Figure $2 \mathrm{~d}$ ) and completely attenuated the ability of Sph-1-P to increase TER (100\% inhibition), consistent with $\mathrm{G}_{\mathrm{i} \alpha}$ coupling to the Edg receptor, which confers the barrier-enhancing response (Figure $2 \mathrm{~d}$ ). We next used two transfection strategies to further characterize signaling events driven by Edg receptor ligation. First, endothelial cells were transduced with a minigene plasmid that encodes an 11 mer that effectively uncouples receptors from $\mathrm{G}_{\mathrm{i} \alpha 1 / 2}$-mediated signaling $(27,33)$. In these experiments, whereas Sph-1-P produced dramatic 0.3 - to 0.5 -unit increases in TER (vector alone), the magnitude of this increase was partially reduced in cells transfected with the $\mathrm{G}_{\mathrm{i} \alpha 1 / 2}$ minigene ( $40 \%$ inhibition) (Figure $2 d$ ), consistent with a role for $G_{i \alpha 1 / 2}$ in the endothelial cell barrier protective response to Sph-1-P. Transfection with either $\mathrm{G}_{\mathrm{s}}$ - or $\mathrm{G}_{\mathrm{q}}$-specific minigenes failed to alter Sph-1-P-induced TER (data not shown) and the $G_{i \alpha 1 / 2}$ minigene failed to alter the TER decline evoked by ligation of the proteolytically activated thrombin receptor whose reductions in TER do not involve $G_{i \alpha}$ coupling (data not shown) (37). Finally, transient transfection of a gene expressing the $\beta$ ARK peptide that retards $\beta \gamma$ subunit signaling by competing for $\beta \gamma$ subunits, also partially attenuated Sph-1-P-induced TER alterations ( $\sim 20 \%$ inhibition) (Figure $2 \mathrm{~d})$. Together, these results are strongly indicative of a signaling pathway that involves Sph-1-P ligation of Edg receptors coupled to $\mathrm{G}_{\mathrm{i} \alpha}$, but do not exclude additional stimulus/coupling mechanisms, which lead to endothelial cell barrier enhancement.

Regulation of Sph-1-P-induced barrier protection by specific signaling pathways. Signal transduction via MAP kinases plays a key role in a variety of cell functions, including several models of endothelial cell permeability (38). We demonstrated recently that $\mathrm{Sph}-1-\mathrm{P}$ rapidly increases the activity of the p42/p44 (ERK) and p38 MAP kinases in lung endothelium by ligation of a PTX-sensitive receptor, presumably Edg-1 (27). To examine whether MAP kinase activation is involved in Sph-1-P-induced barrier protection, endothelial cell monolayers were pretreated with pharmacologic inhibitors of either ERK kinase (MEK) (UO126) or p38 MAP kinase (SB203580). Inhibition of each MAP kinase pathway failed to alter the ability of Sph-1-P to enhance endothelial cell TER (Figure 3) under conditions that significantly reduce PMA-mediated declines in TER as noted previously (38). Similarly, the phosphatidylinositol PI-3'-kinase inhibitor LY294002, or the $\mathrm{p}^{\mathrm{src}}$ inhibitor PP2, had no effect on Sph-1-P-mediated increases in TER (Figure 3), consistent with their failure to inhibit endothelial chemotaxis (27). In contrast, treatment of endothelial monolayers with the generalized tyrosine kinase inhibitors genistein (but not its inactive a
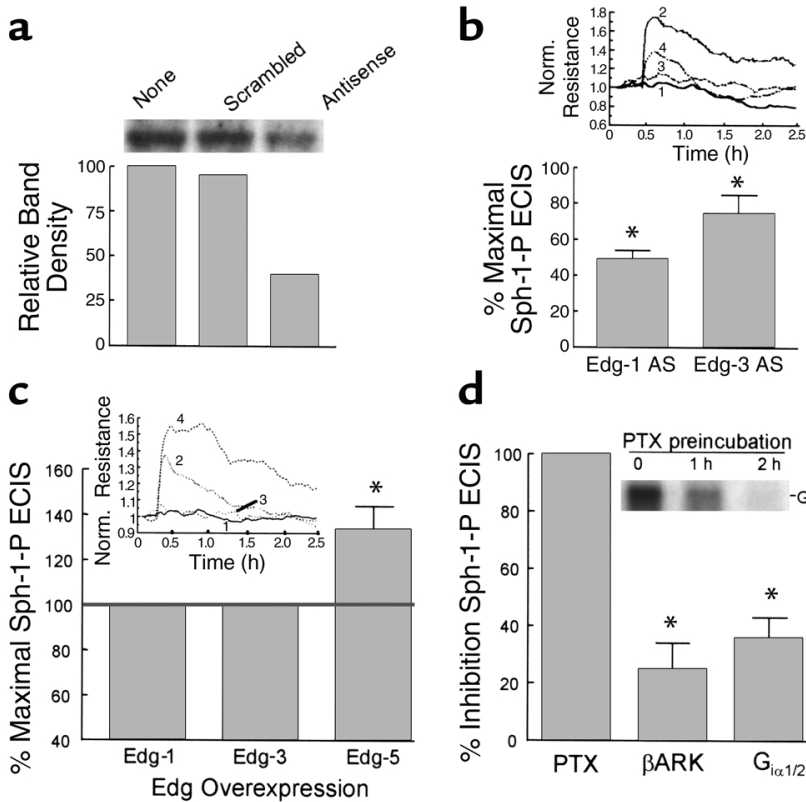

d

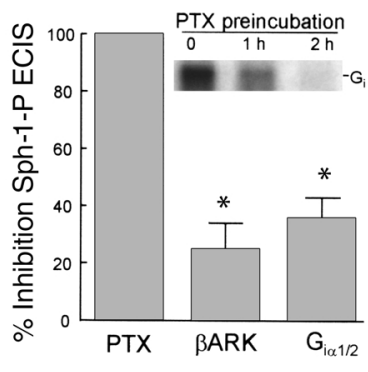

\section{Figure 2}

Role of Edg family receptors and heterotrimeric G proteins in Sph-1P-mediated endothelial cell barrier regulation. (a) Subconfluent bovine endothelial cells were incubated with Edg-1 antisense or their respective control scrambled oligonucleotides $(10 \mu \mathrm{g} / \mathrm{ml}, 48$ hours $)$. This treatment reduced Edg expression by approximately $50 \%$, whereas control oligonucleotide was without effect. (b) In similar experiments, Edg-1 and Edg-3 antisense (AS) oligonucleotides significantly attenuated the Sph-1-P-induced TER response. Sph-1-P-induced TER increased from baseline normalized resistance level of 1.0 to a level of 1.5 to 1.75 in control oligo-treated cells. Data are presented as percentages of Sph-1-P-induced TER in AS-treated cells to Sph-1$\mathrm{P}$-induced TER in control oligonucleotide-treated cells (mean $\pm \mathrm{SE}$, $n=3, P<0.05)$. Inset: ECIS tracing reflecting the reduction of the Sph-1-P response in Edg-1-AS treated cells. Tracing number 1, cells with control oligo; number 2, cells with control oligo and stimulated with Sph-1-P; number 3, cells with Edg-1 antisense; and number 4, cells with Edg-1 antisense and stimulated with Sph-1-P. (c) Bovine endothelial cells were cotransfected with pEGFP and a plasmid encoding either Edg-1, Edg-3, Edg-5, or the empty vector. GFP-positive cells were isolated by flow cytometry (13) and subjected to TER measurements. The percentage of changes of resistance in Edg-transfected cells relative to the vector-transfected cells were calculated. Overexpression of Edg-5, but not Edg-1 or Edg-3, augmented Sph-1$\mathrm{P}$-induced increases in TER. Data are presented as percentage of Sph-1-P-induced TER in cells overexpressing Edg receptors to Sph-1$\mathrm{P}$-induced TER in cells transfected with vector control (mean $\pm \mathrm{SE}$, $n=3, P<0.05)$. Inset: ECIS tracing to show the augmentation of Sph-1-P response in cells expressing Edg-5. In tracing number 1 cells are transfected with the empty vector; in tracing number 2 cells are transfected with the empty vector and stimulated with Sph-1-P; in tracing number 3 cells are transfected with Edg-5; and in tracing number 4 cells are transfected with Edg-5 and stimulated with Sph-1-P. (d) Bovine endothelium were either incubated with PTX $(200 \mathrm{ng} / \mathrm{ml}$, 2 hours) or transfected with plasmids encoding G protein inhibitory peptides $\beta$ ARK or $G_{i \alpha 2}$ using pEGFP cotransfection and cell-sorting strategies described for $\mathbf{c}$. Each G protein manipulation, that is, PTX, $\beta A R K$, and $G_{i \alpha 1 / 2}$ expression, significantly attenuated the Sph-1-P response (mean $\pm \mathrm{SE}, n=3, P<0.05$ ). Inset: Time-dependent ADP ribosylation of $G$ protein in bovine endothelial monolayers incubated with PTX followed by in vitro ADP-ribosylation. PTX treatment before cell lysis completely inactivated $G_{i}$ protein via ADP-ribosylation as we have described previously $(27,28)$. 
a

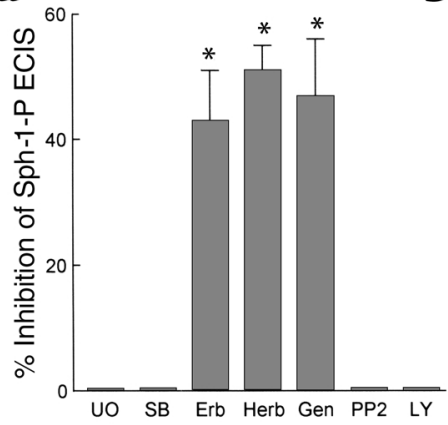

b

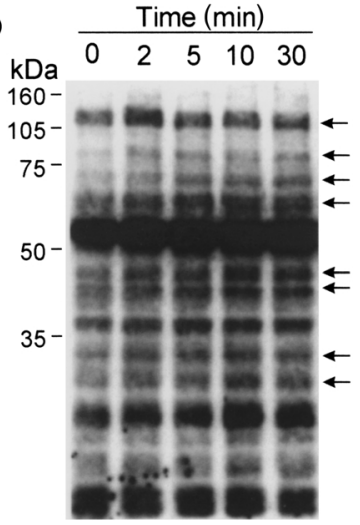

\section{Figure 3}

Signaling events involved in Sph-1-P-mediated endothelial cell barrier enhancement. (a) Bovine endothelial cells were incubated with tyrosine kinase inhibitors genistein (Gen; $100 \mu \mathrm{M}, 30$ minutes), herbimycin A (Herb; $5 \mu \mathrm{M}, 8$ hours), or erbstatin analog (Erb; $20 \mu \mathrm{M}, 1$ hour); the PI-3' kinase inhibitor LY294002 (LY; $25 \mu \mathrm{M}, 30$ minutes), p42/p44 MAP kinase inhibitor UO126 (UO; $10 \mu \mathrm{M}, 30$ minutes), the p38 MAP kinase inhibitor SB203580 (SB; $20 \mu \mathrm{M}, 30$ minutes), or p60 src inhibitor PP2 $(10 \mu \mathrm{M}, 30$ minutes), followed by stimulation with Sph-1-P $(1 \mu \mathrm{M})$. TER was continuously measured for 3 hours and the maximal Sph-1-P response (10-15 minutes after stimulation) were employed for determining the effect of the inhibitors on Sph-1-P-induced barrier enhancement. Data are presented as mean $\pm \mathrm{SE}, n=3, P<0.05$. Genistein, herbimycin $A$, and erbstatin analogues did not significantly alter the basal TER, but inhibited Sph-1-P-induced increases in TER, indicating the involvement of tyrosine kinases in Sph-1-P-mediated modulation of endothelial cell barrier integrity. (b) Endothelial cells were incubated with Sph-1-P $(1 \mu \mathrm{M})$ for indicated times, followed by Western analysis using an phosphotyrosine Ab. Sph-1-P stimulated tyrosine phosphorylation of multiple proteins as indicated by arrows.

analogue), herbimycin, and erbstatin, significantly reduced Sph-1-P-evoked increases in TER (Figure 3a), findings supported by the increase in endothelial phosphotyrosine proteins after Sph-1-P (Figure 3b). These results reinforce our hypothesis that $\mathrm{Sph}-1-\mathrm{P}$ enhances barrier integrity and reverses established barrier dysfunction via specific cellular signaling pathways potentially involved in the final steps of the angiogenic response.

Sp1-P induces endothelial cell cortical cytoskeletal rearrangement. The endothelial cell cytoskeleton is a critical determinant of vascular integrity and barrier regulation (39-41) and known to be influenced by tyrosine phosphorylation (32). We examined the effect of Sph-1-P on the spatial localization and polymerization of actin and myosin by immunofluorescent microscopy. Consistent with the observed increase in endothelial cell monolayer integrity reflected by transmonolayer electrical resistance, Sph-1-P $(1 \mu \mathrm{M})$ produced rapid and dramatic enhancement of polymerized F-actin and myosin heavy chain A staining that was spatially confined to the cortical cytoskeletal ring (Figure 4). These findings occurred in conjunction with evidence of lamellipodia formation, but without the appearance of increased stress fibers or paracellular gaps, features evoked by edemagenic agents such as thrombin (Figure 4). To significantly link actin cytoskeletal changes to the physiologic effects of Sph-1-P, we employed three complementary approaches. First, we pretreated human endothelial cell monolayers with the actin depolymerizing agent cytochalasin $\mathrm{B}$, which produced a prompt decline in TER (Figure 5a). Unlike the protective response to Sph-1-P challenge after established thrombin-induced barrier dysfunction (Figure 1d), Sph-1-P failed to increase TER when added after cytochalasin challenge, indicating a critical requirement for dynamic cytoskeletal rearrangement and an intact actin cytoskeleton for Sph-1-P barrier-enhancing effects (Figure 5a). Secondly, we used latrunculin $B$, which, unlike cytochalasin, does not induce actin depolymerization, but inhibits actin polymerization. Similar to the results observed with cytochalasin, latrunculin reduced basal TER while preventing the prompt increase in TER typically observed after Sph-1-P. Finally, we employed a model of microtubule disruption with nocodazole, a potent cause of barrier disruption produced without actin disassembly. Figure $5 c$ demonstrates that nocodazole failed to alter the Sph-1-P-mediated TER response, indicating the specific requirement for actin dynamic assembly/disassembly in the Sph-1-P-mediated barrier-enhancing response.

\footnotetext{
Figure 4

Effect of receptor agonists on bovine endothelial cell cytoskeletal structure. Cells were treated with either vehicle (a and d), Sph-1-P (1 $\mu \mathrm{M}$ for 5 minutes) (b and $\mathbf{e}$ ), or thrombin (100 nM for 15 minutes) (c and f). F-actin staining was assessed with Texas red phalloidin (a-c) and myosin staining evaluated with antimyosin Ila polyclonal Ab (d-f). Thrombin induces dissolution of the cortical cytoskeleton, prominent stress fiber formation, and formation of intercellular gaps. In contrast, Sph-1-P significantly enhances cortical actomyosin staining, which correlates with enhancement of endothelial cell barrier function.
}
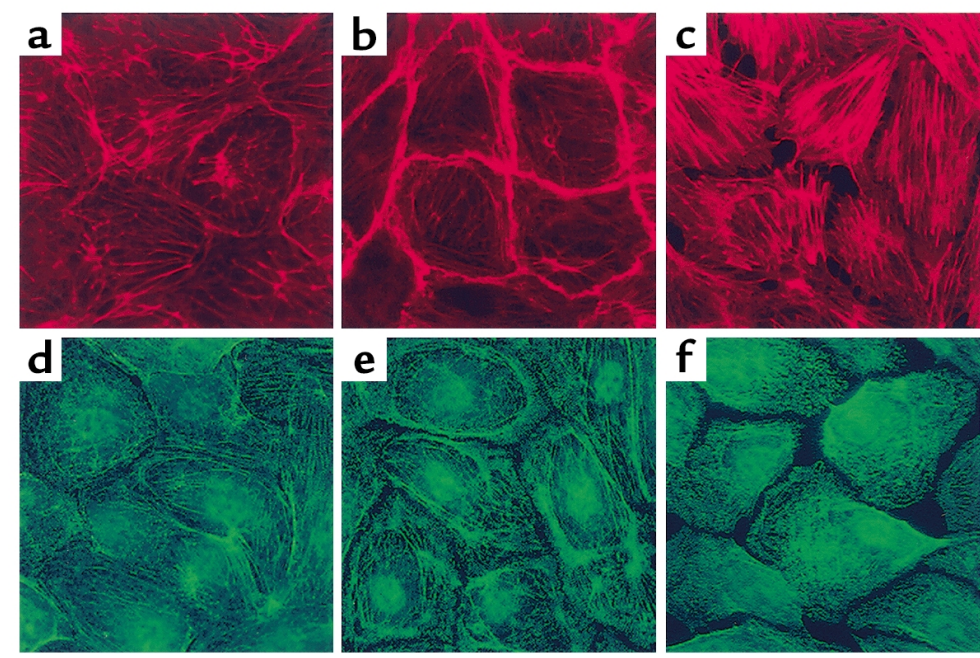

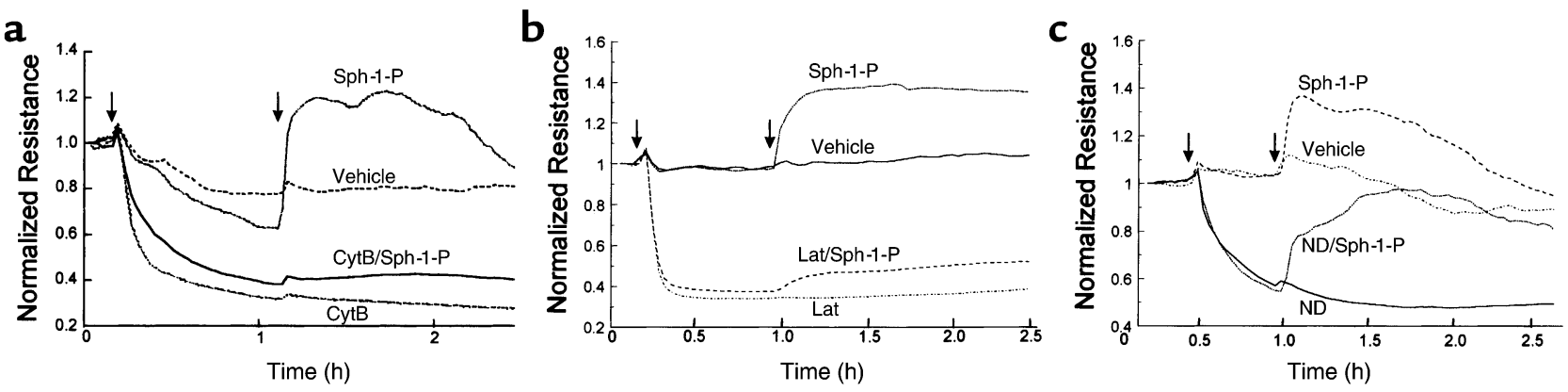

\section{Figure 5}

Effect of cytoskeletal alterations on Sph-1-P-induced endothelial cell barrier protection. (a) Bovine endothelium, grown on gold microelectrodes, was pretreated with either vehicle or cytochalasin B (CytB; $25 \mu \mathrm{g} / \mathrm{ml}, 60$ minutes), followed by stimulation with Sph-1-P (1 $\mu \mathrm{M})$. Actin depolymerization and disruption of microfilament structure decreases TER and completely prevented the effect of Sph-1-P on TER. (b) Human endothelial cells were pretreated with either vehicle or latrunculin A (Lat; $0.1 \mu \mathrm{M}, 40$ minutes), followed by stimulation with Sph1-P $(1 \mu \mathrm{M})$. Inhibition of actin polymerization decreases basal level TER and prevented Sph-1-P-mediated TER. (c) Bovine endothelial cells were incubated with either vehicle or the microtubule-disrupting agent, nocodazole (ND; $2.5 \mu \mathrm{M}, 30$ minutes), followed by stimulation with Sph-1-P $(1 \mu \mathrm{M})$. Disruption of microtubule decreases TER, but failed to alter Sph-1-P-induced increases in TER.

We next investigated whether increases in MLC phosphorylation, an event associated with the synergistic action of MLC kinase (MLCK) and Rho kinase, participate in the enhanced TER measurements induced by Sph-1-P. Immunofluorescent staining with antisera specific for MLCs that have been phosphorylated on Ser 19 revealed a clear increase in phospho-MLC immunoreactivity in confluent and subconfluent cells, which was restricted to the dense peripheral band after Sph-1-P challenge when compared with controls. In contrast, phospho-MLC staining was profound after thrombin challenge but observed in the prominent cytosolic stress fibers formed (Figure 6), suggesting the spatially defined and peripherally distributed increases in MLC phosphorylation after Sph-1-P may not be catalyzed by increased MLCK activity. Consistent with these results and studies of Sph-1-P-induced endothelial cell migration (27), both inhibition of MLCK activity with ML-7 (20 $\mu \mathrm{M}, 30$ minutes) (Figure 7$)$ and pretreatment with BAPTA-AM to chelate intracellular $\mathrm{Ca}^{2+}$ (data not shown), dual processes known to abolish MLCK activity $(41,42)$, failed to alter the Sph-1$\mathrm{P}$-mediated barrier-enhancing responses (Figure 7). The thrombin response, however, was significantly reduced, arguing against the contribution of $\mathrm{Ca}^{2+}$-dependent processes (including MLCK activity) to Sph-1-P-mediated barrier regulation. Figure 7 also demonstrates the sensitivity of TER measurements to Rho kinase inhibition after either Sph-1-P or thrombin challenge. Consistent with our previous endothelial chemotaxis data (27), two Rho kinase inhibitors, Y27632 (Figure 7) and HA-1077 (data not shown), partially attenuated the barrier protective effects of Sph-1-P $(\sim 30 \%)$. We further noted that the increase in cortical actin that persisted for up to 2 hours after Sph-1-P challenge was only marginally affected by Rho kinase

\section{Figure 6}

inhibition, although the level of cytosolic stress fibers normally present in unstimulated monolayers was entirely eliminated (data not shown). These results suggest that while Rho kinase actively participates in endothelial cell barrier regulation, Sph-1-P influences endothelial cell cortical actin assembly through Rhoindependent mechanisms that result in localized actomyosin remodeling in a temporal fashion consistent with barrier enhancement.

Role of Rac GTPase and PAK in Sph-1-P-mediated actin rearrangement. Previous studies have implicated the participation of Rac GTPase in lamellipodia formation and cortical cytoskeletal reorganization (43). Indeed, we found Sph-1-P to rapidly activate Rac GTPase (1 minute) in a PTX-sensitive manner (Figure 8a), and transient transfection with a plasmid encoding constitutively active Rac (V12) reproduced changes in the cortical actin similar to those evoked by Sph-1-P (Figure 8, b-g). Immunofluorescent studies further demonstrated that Sph-1-P-induced cortical rearrangement may involve a cytoskeletal signaling sequence that includes the known Rac GTPase target, the p21-associated

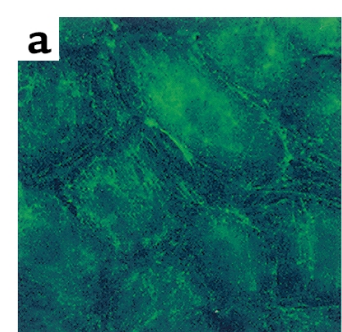

Vehicle

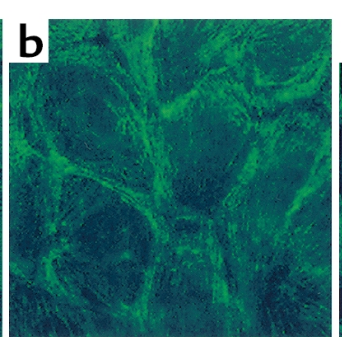

Sph-1-P

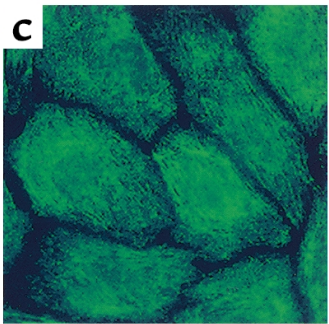

Thrombin

Effect of receptor agonists on bovine endothelial cell MLC phosphorylation. Cells were treated with either vehicle (a), Sph-1-P (1 $\mu \mathrm{M}$ for 5 minutes) (b), or thrombin (100 nM for 15 minutes) (c). Phospho-MLC staining was assessed with specific antimonophospho MLC Ab. Sph-1-P challenge locally increases MLC phosphorylation in the cortical actin ring. In contrast, thrombin significantly enhances phospho MLC staining, which appears to colocalize with cytoplasmic actin stress fibers. 


\section{Figure 7}

Effect of Rho kinase and MLCK inhibition on Sph-1-P-mediated changes in TER. (a) Confluent bovine pulmonary artery endothelium grown on gold microelectrodes were pretreated with either vehicle control, Rho kinase inhibitor, Y27632 $(10 \mu \mathrm{M})$, or the MLCK inhibitor, ML-7 $(20 \mu \mathrm{M})$ for 60 minutes, followed by stimulation with Sph-1-P $(1 \mu \mathrm{M})$ or thrombin (Thr, $100 \mathrm{nM})$. The maximal Sph-1-P-induced endothelial barrier enhancement observed at 10-15 minutes was attenuated by the reduction of Rho kinase activity, but not affected by the inhibition of MLCK activity, whereas maximal thrombin-induced endothelial barrier disruption (30 minutes) was significantly blocked by both Rho kinase and MLCK inhibition. Data are mean $\pm \mathrm{SE}, n=3, P<0.05$.

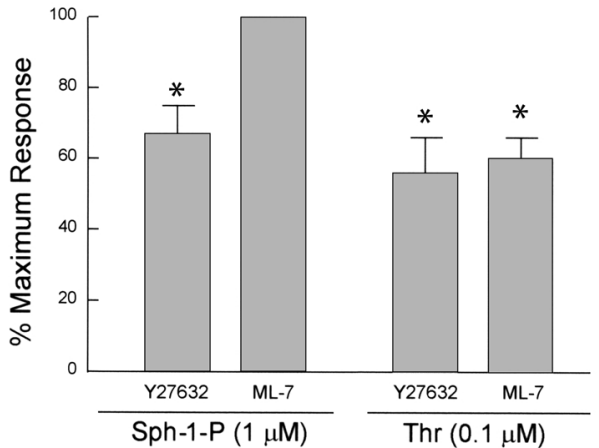

Ser/Thr kinase (PAK), whose binding of Rac results in the phosphorylation and activation of LIM kinase and the subsequent inactivation of the LIM kinase target, cofilin, an actin-severing protein (44). Figure 9 demonstrates the rapid translocation of both PAK and cofilin to the cortical actin cytoskeleton after Sph-1-P challenge. More importantly, transient transfection of human endothelium with a PAK-1 dominant negative construct dramatically reduced Sph-1-P-induced increases in the cortical distribution of polymerized actin as compared with vector alone (Figure 10) and abolished cofilin translocation (data not shown). We next evaluated the role of PAK/LIM kinase in Sph-1$\mathrm{P}$-induced actin rearrangement response by overexpressing the actin depolymerizing factor known as cofilin, a recognized substrate for PAK/LIM kinase phosphorylation. Adenoviral-mediated cofilin overexpression did not alter baseline electrical impedance, but significantly blunted the barrier-enhancing effects of Sph-1-P (Figure 11). Immunofluorescence studies revealed a more poorly developed cortical actin ring in Sph-1-P-challenged cells transfected previously to overexpress wild-type cofilin compared with nontransfected cells or cells transfected with empty vector. Together, these studies indicate the critical role for the cortical actin rearrangement in Sph-1-P-mediated endothelial cell barrier integrity.

\section{Discussion}

The maintenance of the normal endothelial cell barrier and the integrity of the microcirculation is a critical component of fluid and protein homeostasis and a terminal event of new blood vessel formation. This process involves platelets and their products that preserve capillary integrity, although the mechanism of this barrier protective action has not yet been precisely elucidated. $\mathbf{a}$

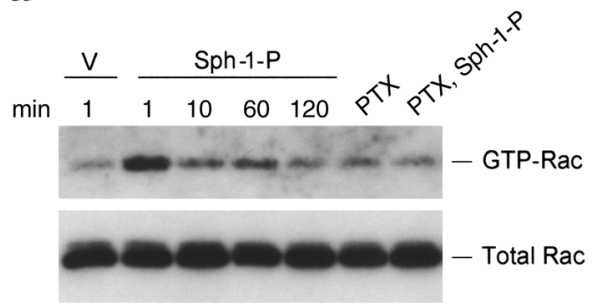

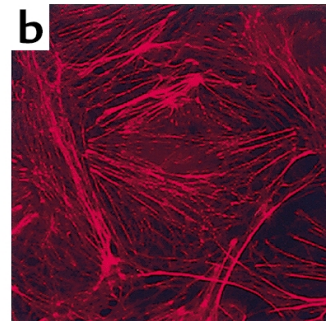
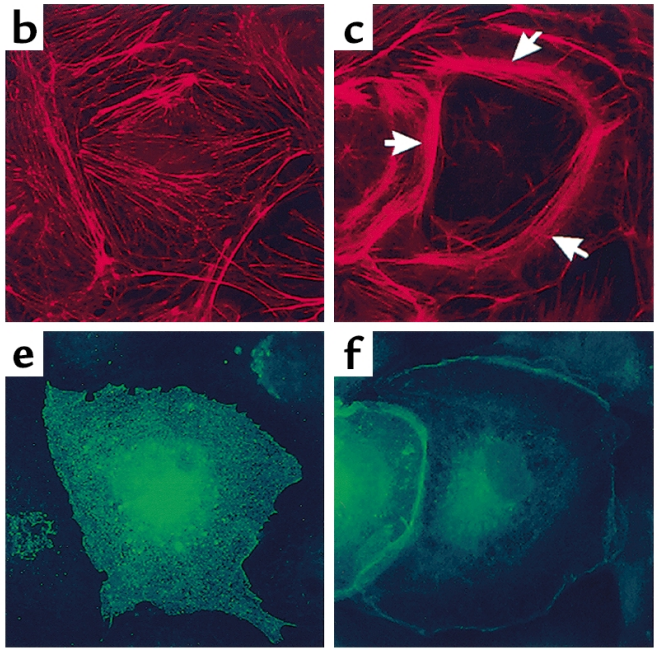
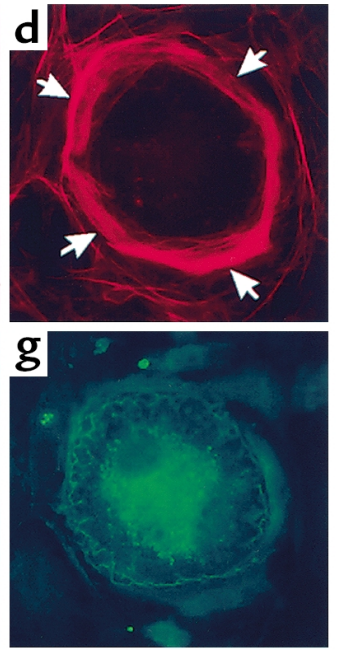

\section{Figure 8}

Role of Rac GTPase in Sph-1-P-mediated endothelial actin rearrangement and barrier regulation. (a) Bovine pulmonary artery endothelium were incubated with vehicle $(v)$ Sph-1-P $(1 \mu \mathrm{M})$, for indicated periods of time, or pretreated with PTX $(1 \mu \mathrm{g} / \mathrm{ml}, 2$ hours $)$ and subsequently incubated with Sph-1-P for 1 minute. Cells were lysed, centrifuged, and supernatants were collected. The activated GTP-bound Rac was precipitated by agarose-conjugated human PAK-1 p21-binding domain and immunoblotted by anti-Rac mAb as described in Methods. Total Rac content was detected using cell lysates. These results indicate rapid Sph-1-P-induced Rac activation, which was completely abolished by PTX. (b-g) Bovine endothelium was transfected with either an empty vector ( $\mathbf{b}$ and $\mathbf{e}$ ) or a constitutively active HA-tagged Rac construct $(\operatorname{Rac} \mathrm{V} 12)(\mathbf{c}, \mathbf{d}, \mathbf{f}$, and $\mathbf{g})$ as described in Methods. Shown are subsequent immunofluorescence images $(\times 100)$ of endothelial cells stained with either Texas red phalloidin for F-actin (b-d) or anti-HA tag Ab for identification of transfected cells $(\mathbf{e}-\mathbf{g})$. $\mathbf{b}$ and $\mathbf{e}, \mathbf{c}$ and $\mathbf{f}, \mathbf{d}$ and $\mathbf{g}$ represent matched images. Overexpression of constitutively active Rac V12, but not control vector, significantly enhances polymerized actin staining (arrows) within the cortical ring with the degree of enhancement dependent upon the level of Rac V-12 overexpression. 


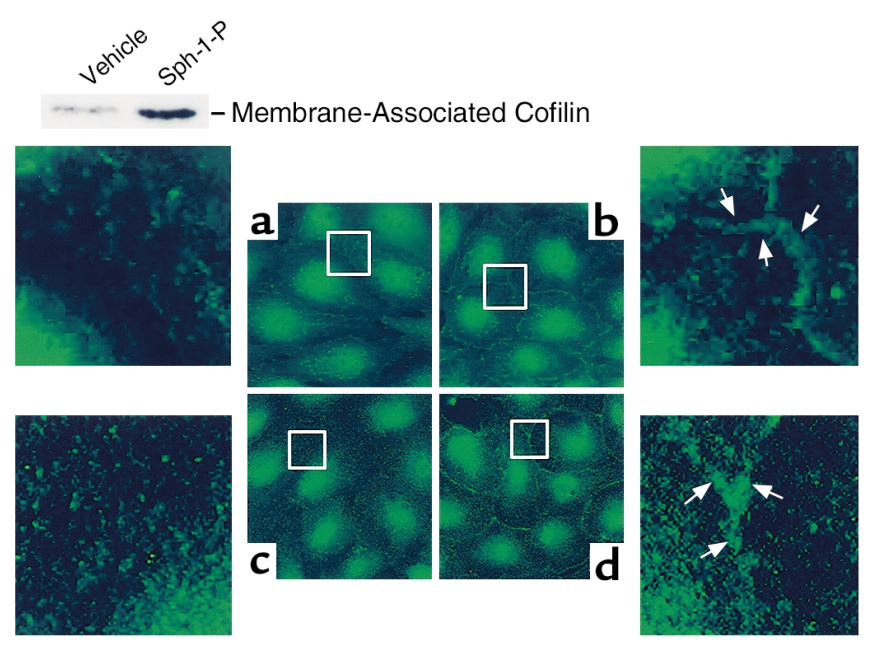

\section{Figure 9}

Effect of Sph-1-P on cofilin and PAK subcellular localization. Human endothelial cells were treated by either media (two left panels), or Sph-1-P (two right panels) for 5 minutes. PAK-1 staining (a and $\mathbf{b}$ ) was assessed with specific anti-PAK $1 \mathrm{Ab}$ as described in Methods. Cofilin staining ( $\mathbf{c}$ and $\mathbf{d}$ ) was assessed with specific anti-cofilin Ab. Sph-1-P caused rapid translocation of both cofilin and PAK-1 to the cell periphery (translocated proteins shown by arrows). Inset: Western blot demonstrating increased cofilin content in the membrane fraction. Human endothelial cells were challenged with vehicle or Sph-1-P for 5 minutes. Cell lysates then underwent detergent fractionation and the membrane and cytosol fractions $(10 \mu \mathrm{g} /$ lane $)$ separated by SDS PAGE and stained with anti-cofilin Ab.
We were the first to report a direct effect of plateletderived phospholipids, such as Sph-1-P, on endothelial cell activation (45). However, since that report, much attention has been directed to defining the potential role of platelet-released phospholipids on vascular integrity and angiogenesis. We reported that PA directly perturbed endothelial cell barrier function (11) and began to extend our interest to other platelet products with potentially vasoactive properties. Alexander and Haselton first demonstrated (7), and we confirmed (8), that LPA has a weak but consistent enhancing effect on endothelial cell barrier integrity in vitro. This effect of LPA may be tissue specific since LPA has been noted to increase the permeability of brain microvessels (10). LPA and Sph-1-P are structurally related lysophospholipids, which have the capacity to evoke similar physiological and pathophysiological responses (26). We reported recently that Sph-1-P is a potent lung endothelial cell chemoattractant and the primary angiogenic factor present in serum where it is generated by platelets during clotting $(12,27)$. Sph-1-P directly induces angiogenesis in avian chorion (6) while potentiating peptide growth factor-mediated angiogenesis in vivo (14), including the avascular mouse cornea model (12). The effect of Sph-1-P on endothelial cell permeability, an activity shared by several angiogenic agents, has not been reported previously.

Our results demonstrate that Sph-1-P is a potent and direct endothelial monolayer- stabilizing agent that can reverse barrier disruption induced by edemagenic agents such as thrombin. Thus, while Sph-1-P is found in low levels in blood plasma, its release into serum during clotting allows it to participate in angiogenesis and in the critical development of functional integrity of newly formed blood vessels. Recently, Edg-1-deficient mouse embryos were noted to display significant abnormalities in vascular development, rendering the newly formed vessels leaky, resulting in fluid extravasation and hemorrhage (18). Our data are consistent with the attractive hypothesis that Sph-1-P directly impinges upon newly formed cells to increase barrier properties, thereby abolishing vascular leak and the resultant edema, a property recently described for angiopoietin-1 (19), while promoting vessels to mature normally. We have attempted to provide preliminary information regarding the signaling mechanisms by which Sph-1-P accomplishes barrier protection and to identify potential downstream mediators, since these may be useful
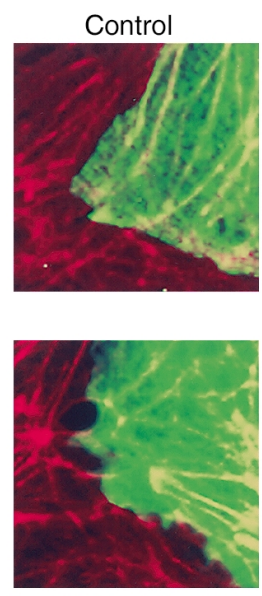

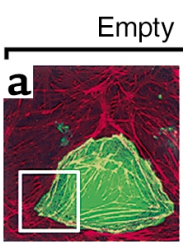

mpty Vector
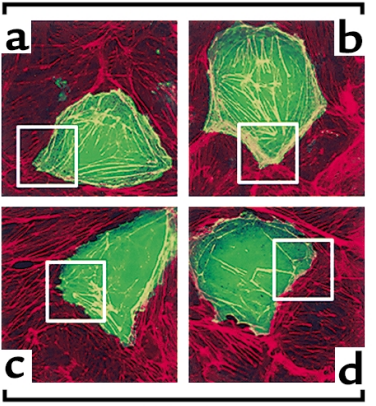

PAK D/N
Sph-1-P

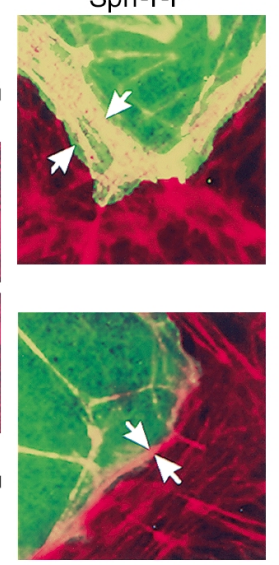

\section{Figure 10}

Effect of PAK inhibition on Sph-1-P-induced endothelial cell cytoskeletal rearrangement. Bovine endothelial cells were transfected with either empty vector ( $\mathbf{a}$ and $\mathbf{b}$ ) or dominant negative c-myc-tagged PAK-1 construct (c and $\mathbf{d}$ ) as described in Methods, followed by challenge with either media (a and $\mathbf{c}$ ) or $1 \mu \mathrm{M}$ Sph-1-P (b and $\mathbf{d}$ ) for 5 minutes. Shown are subsequent merged immunofluorescence images $(\times 100)$ of endothelial cells stained with Texas red phalloidin for F-actin (red) and anti-c-myc tag Ab for identification of PAK-1 overexpressing cells (green). Overexpression of dominant negative PAK-1 but not empty vector significantly inhibited Sph-1-P-induced actin cortical ring enhancement (F-actin staining in transfected cells after merging green and red images appears as yellow). Arrows point to the increased cortical actin band in the Sph-1-P-treated cells. 

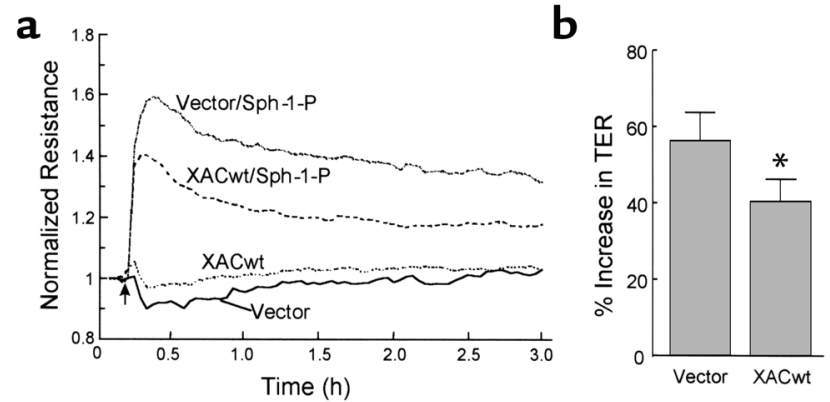

\begin{abstract}
Figure 11
Cofilin overexpression significantly attenuates Sph-1-P-mediated increases in TER. Approximately $80 \%$ confluent human endothelial cells grown on gold microelectrodes were infected with recombinant adenovirus expressing a GFP-tagged wild-type xenopus ADF/cofilin (XACwt) or vector control that expresses GFP only. Cells were cultured in complete medium for 72 hours, followed by stimulation with Sph-1-P $(1 \mu \mathrm{M})$. Shown is a representative tracing. Although basal TER was unaffected by cofilin overexpression, the increases in TER (percentage over basal) stimulated by Sph-1$P$ were significantly attenuated in the XACwt virus-infected cells. (b) The results pooled from four independent experiments. Data represent TER values obtained 10-15 minutes after Sph-1-P addition (mean $\pm \mathrm{SD}, P<0.05$ ).
\end{abstract}

therapeutic targets in the regulation of the angiogenic responses and barrier dysfunction. Our data addressing barrier regulation are consistent with the literature that most in vitro and in vivo angiogenic activities elicited by Sph-1-P are inhibited by Edg-1 and Edg-3 antisense oligonucleotides $(14,15)$. Antisense oligonucleotides directed at Edg-1 and, to a lesser degree, Edg-3, reduced but did not abolish Sph-1-P-mediated enhanced TER, which we believe reflects incomplete reduction in Edg- 1 and Edg- 3 expression by our antisense strategies (Figure 2a) (27). Because Edg-5 expression was not detected (27), overexpression of Edg-5, but not Edg-1 and Edg-3, would be expected to increase TER if all three receptors are linked to the signaling pathway that leads to vascular integrity potentiation. The role of Edg-5 in the angiogenic and chemotactic responses remains questionable. However, given the remarkable effect of Edg-5 overexpression on endothelial cell barrier protection (Figure 2c), it would be of interest to more fully evaluate the role of Edg-5 in angiogenesis as a receptor that may be transiently expressed in vivo during critical phases of blood vessel formation.

Although Edg receptors have been variably described as coupled to three heterotrimeric $G$ proteins, $G_{i}$, $\mathrm{G}_{12 / 13}$, and $\mathrm{G}_{\mathrm{q}}(26,46)$, several lines of evidence indicate that Sph-1-P signals involved in endothelial barrier function are transduced primarily via $E d g-G_{i \alpha}$ coupling. Sph-1-P-mediated increases in endothelial cell TER were completely abolished by PTX and reduced by complementary molecular strategies to interrupt $G$ protein signaling (specific $\mathrm{G}_{\mathrm{ia} 1 / 2}$ and $\beta A R K$ minigenes). These results are particularly intriguing when coupled with our previous report indicating that $\mathrm{G}_{i \alpha}$ inhibition by PTX directly increases endothelial cell permeability and intercellular gap formation while reducing TER via intrinsic ADP ribosyltransferase activity (47). Given the prominent role we now demonstrate for Sph-1P-Edg coupling in barrier regulation and its ability to reverse thrombin-mediated permeability (Figure 1), it is tempting to speculate that the stimulus/coupling sequence of Sph-1-P-Edg- $\mathrm{G}_{\mathrm{i} \alpha}$, occurring under homeostatic conditions or at sites of localized coagulation, may represent a physiologically relevant, barrier-protective signaling pathway triggered as a consequence of hemostasis and platelet aggregation. Although Edg receptors are linked to tyrosine kinase activities such as $\mathrm{p} 60^{\mathrm{src}}$ and Sph-1-P has been reported to activate p60 $0^{\text {src }}$ in a PTX-dependent fashion $(26,46)$, this barrier-enhancing pathway does not appear to involve MAP kinases or $\mathrm{p} 60^{\mathrm{src}}$ (Figure 3 ), findings remarkably similar to our studies of Sph-1-P-mediated endothelial cell migration (27). In contrast, generalized tyrosine kinase inhibitors such as genistein, herbimycin, and erbstatin each produced a significant reduction in Sph-1P-enhanced TER response (Figure 3).

Our data strongly suggest the critical participation of active actin cytoskeletal rearrangement linked to increased extracellular matrix tethering in the barrier protection induced by Sph-1-P (Figure 4 and 5). Our recent results confirm the critical role of Rho family GTPases in regulating the cytoskeleton (Rho and Rac) and confirm their critical participation in endothelial chemoattractant responses (27). Strategies to reduce Rho activities also decreased Sph-1-P-stimulated endothelial cell chemotaxis, whereas the migration response to VEGF was not affected, suggesting both overlapping and distinct pathways for accomplishing cell locomotion (27). Rho GTPase, while involved in endothelial cell migration, does not appear to be the sole mechanism involved in either Sph-1-P-mediated cortical cytoskeletal rearrangement or barrier-protective responses. Rho kinase inhibition produced only partial $(\sim 30 \%)$ attenuation of Sph-1-P-mediated increases in TER. Sph-1-P-mediated tyrosine phosphorylation of the cytoskeletal docking protein Crk-associated substrate, Cas, occurred in a Rho kinase-independent manner (48), whereas tyrosine phosphorylation of focal adhesion kinase (FAK) occurred in endothelium in a Rho kinasedependent fashion (49). Further studies are required to evaluate the exact role of Rho kinase in the barrierenhancing cytoskeletal signals evoked by Sph-1-P.

An important aspect of our work is defining a role of the cortical cytoskeleton in the novel Sph-1-P-mediated barrier enhancement. Regulation of this response is complex and, as noted above, is quite different from migration regulation. Our results support a role for the Rac GTPase in the remarkable enhanced integrity of the endothelial cytoskeleton and barrier function after Sph-1-P. Rac was rapidly activated and molecular manipulations of Rac V12 GTPase activity reproduced Sph-1-P-induced increases in actin rearrangement (Figure 8). Our previous studies, but not all 
reports (50), did not demonstrate that Sph-1-P produces biochemical evidence of generalized increases in bovine pulmonary artery endothelial cell MLC phosphorylation (27), and inhibition of the novel endothelial cell MLCK isoform (42) did not alter either Sph-1$\mathrm{P}$-induced chemotaxis (27) or protective TER responses (Figure 7). However, as shown in Figure 6, there were clear spatially defined increases in Ser 19 phosphorylation within the cortical actin ring after Sph-1-P. Recently, several kinases, in addition to MLCK, have been described to phosphorylate MLC on Ser 19 in vitro, including Rho kinase (51) and PAK (52). We believe our immunofluorescent data provides convincing evidence that PAKs, a class of Rac-dependent Ser/Thr kinases (53) directly stimulated by sphingosine (54), functions to modulate the activity of the endothelial actin-myosin cytoskeleton, to increase cortical actin, and to enhance endothelial cell barrier properties. PAK was recently described to phosphorylate smooth muscle MLCK, thereby inhibiting MLCK activity (55); however, whether this mechanism is operative for the endothelial cell MLCK isoform closely regulated by Ser/Thr (42) and Tyr phosphorylation (56) is not known. Although actin assembly/disassembly is a very complex process, consistent with reports of PAK localization at cortical actin structures in growth-stimulated cells (57), we demonstrate that Sph-1-P increases immunoreactivity in the cortical actin ring of PAK, the Ser/Thr kinase known as LIM kinase (data not shown), and the actin-binding protein, cofilin. PAK dominant negative constructs completely abolished Sph-1-P-mediated actin rearrangement (Figure 10) as well as cofilin translocation to the cortical actin ring (data not shown). Unphosphorylated cofilin severs and depolymerizes actin filaments in vitro, promoting rapid actin filament turnover in vivo (58-61), whereas phosphorylation of cofilin at Ser 3 catalyzed by LIM kinase, a PAK phosphorylation target, reverses cofilin-induced actin depolymerization and leads to accumulation of actin filaments $(44,61$, 62 ). We overexpressed the LIM kinase target, cofilin, in endothelial monolayers and subsequently analyzed barrier properties by measuring electrical impedance. Cofilin overexpression did not alter baseline permeability, but blunted the barrier-enhancing effects of Sph-1-P treatment and produced a more poorly developed cortical actin ring in Sph-1-P-challenged cells compared with nontransfected cells or cells transfected with either empty vector or the A3 mutant. We hypothesize that the overexpression of wild-type cofilin upsets the essential balance of F-actin polymerizing and depolymerizing factors necessary for critical cortical actin rearrangement and subsequent permeability changes. These results are consistent with previous studies of the effects of wild-type cofilin and the A3 cofilin mutant on enhanced neurite outgrowth, where wild-type cofilin expression markedly increased growth rates (40-60\%) while the A3 mutant gave only slightly enhanced outgrowth, which was not statisti- cally significant over that of controls (63). We hypothesize that the ability to phosphorylate and dephosphorylate cofilin is an important property in regulating their ability to enhance actin turnover (i.e., phosphocycling of AC). Since the A3 protein cannot participate in phosphocycling, it is less effective in promoting actin dynamics, although it can promote depolymerization of filaments and increase the sequestered monomer pool. Thus, under normal conditions Sph-1-P would stimulate the Rac pathway and activate LIM kinase via PAK, resulting in a net increase in actin polymerization dynamics-if the phosphatase pathway was coordinately activated-or a net phosphorylation of AC leading to an increase in assembled F-actin and net decrease in actin dynamics. If the latter occurred, then overexpressing wild-type cofilin could restore the increase in actin turnover and defeat the net effects of Sph-1-P. These new results show a critical role for cortical actin rearrangement in Sph-1P-induced endothelial barrier function.

The observation that Sph-1-P has profound effects on seemingly divergent biologic responses, for example, enhancing both cell migration as well as monolayer integrity, is interesting. We speculate that the cytoskeletal response to Edg ligation exhibits a degree of specificity depending upon whether cells are in confluent monolayers or in a promigratory, loosely adherent phenotype. We have found that chemotaxis increases if endothelial cells are allowed to adhere to the substratum before exposure to the chemoattractant, suggesting that perhaps, adherence may be a fundamental component of the chemotactic response of endothelial cells.

However, it is possible that Sph-1-P chemotaxis-promoting characteristics and adherence-promoting characteristics are driven by the same basic mechanism. While agents that increase endothelial cell permeability are somewhat chemotactic (e.g., VEGF), their response pales when compared with that induced by Sph-1-P (27). It is conceivable, and perhaps likely, that adherence plays a fundamental role in the chemotactic response. Of potentially greater importance is the fact that Sph-1-P very effectively promotes both migration and barrier integrity, two key aspects of the angiogenic response. Thus, vessels formed in response to Sph-1-P may be less "leaky" than those formed in response to VEGF or other angiogenic factors. Our previous studies (12), and other seminal work (14), demonstrate that Sph-1-P promotes angiogenesis optimally in conjunction with protein growth factors. The exact role of each individual factor in the response, and indeed the identity of all the factors involved in the response, have not been clarified, but an endothelial cell chemoattractant that promotes vascular integrity may certainly play a key role in the response.

In summary, we have demonstrated that Sph-1-P, a recognized potent endothelial cell agonist and angiogenic factor, directly contributes to the maintenance of the integrity of the vascular endothelium. Acting 
through paracrine, autocrine, or juxtacrine pathways, Sph-1-P mediates this important response through Edg receptors that are coupled by a PTX-sensitive G protein to downstream effectors that include Rho and Rac GTPases. Our results suggest that Edg receptor ligation results in a spatially defined, barrier-regulatory signaling cascade that may involve Rac GTPase, PAK-1, and LIM kinase translocation and activation, and the subsequent phosphorylation of cofilin, which reduces actin severing, thereby allowing cortical actin thickening and lamellipodia formation. While our signaling studies are preliminary, they remain secondary to the main finding of this study: that a biologically active mediator released from activated platelets has a dramatic effect on the permeability function of the vascular endothelium. Sph-1-P may be added to a growing list of key endothelial cell growth factors that regulate vascular function and homeostasis and to a very select list of agonists that promote the integrity of the vascular bed. The participation of this novel, biologically active lipid in platelet-endothelial cell interactions may be critical to understanding the complexity of coagulation, wound healing, and angiogenesis, as well as pathogenic macrovascular damage.

\section{Acknowledgments}

This work was supported by grants from the National Heart, Lung, and Blood Institute (HL 50533, HL 58064, HL 69340, and HL 61751) and the Dr. David Marine Endowment. The authors gratefully acknowledge the contributions of Lakshmi Natarajan, Saule Nurmukhambetova, Keri N. Jacobs, and Peiyi Wang for superb technical assistance, and Ellen G. Reather for expert manuscript preparation. Special appreciation is extended to M. Nicolic, Michael T. Crow, W. J. Koch, and N. Aniellin for providing key reagents for this work.

1. Gimbrone, M.A., Jr., et al. 1969. Preservation of vascular integrity in organs perfused in vitro with a platelet-rich medium. Nature. 221:33-36.

2. Roy, A.J., and Djerassi, I. 1972. Effects of platelet transfusions: plug formation and maintenance of vascular integrity. Proc. Soc. Exp. Biol. Med. 139:137-142.

3. Shepro, D., Welles, S.L., and Hechtman, H.B. 1984. Vasoactive agonists prevent erythrocyte extravasation in thrombocytopenic hamsters. Thromb. Res. 35:421-430.

4. Lo, S.K., Burhop, K.E., Kaplan, J.E., and Malik, A.B. 1988. Role of platelets in maintenance of pulmonary vascular permeability to protein. Am. J. Physiol, 254:H763-H771.

5. McDonagh, P.F., 1986. Platelets reduce coronary microvascular permeability to macromolecules. Am J. Physiol. 251: H581-H587.

6. English, D., Garcia, J.G.N., and Brindley, D.N. 2001. Platelet-released phospholipids link haemostasis and angiogenesis. Cardiovasc Res. 49:588-599.

7. Alexander, J.S., Patton, W.F., Christman, B.W., Cuiper, L.L., and Haselton, F.R. 1998. Platelet-derived lysophosphatidic acid decreases endothelial permeability in vitro. Am. J. Phys. 274:H115-H122.

8. English, D., et al. 1999. Induction of endothelial cell chemotaxis by sphingosine 1-phosphate and stabilization of endothelial monolayer permeability by lysophosphatidic acid, potential mediators of hematopoietic angiogenesis. J. Hematother. Stem Cell Res. 8:627-634

9. Yatomi, Y., et al. 2000. Sphingosine 1- phosphate as a major bioactive lysophospholipid that is released from platelets and interacts with endothelial cells. Blood. 96:3431-3438.

10. Schulze, C., Smales, C., Rubin, L.L., and Staddon, J.M. 1997. Lysophosphatidic acid increases tight junction permeability in cultured brain endothelial cells. J. Neurochem. 69:991-1000.

11. English, D., et al. 1999. Induction of endothelial monolayer permeabil- ity by phosphatidate. J. Cell Biochem. 75:105-117.

12. English, D., et al. 2000. Sphingosine 1-phosphate released from platelets during clotting accounts for the potent endothelial cell chemotactic activity of blood serum and provides a novel link between hemostasis and angiogenesis. FASEB J. 14:2255-2265.

13. Kovala, T.A., et al. 2000. High efficiency transient transfection of endothelial cells for functional analysis. FASEB J. 14:2486-2494.

14. Lee, M.J., et al. 1999. Vascular endothelial cell adherens junction assembly and morphogenesis induced by sphingosine-1-phosphate. Cell. 99:301-312.

15. Wang, G., Van Brocklyn, J.R., Hobson, J.P., Movatagh, S., and Zukowska-Grojec, Z. 1999. Sphingosine 1-phosphate stimulates cell migration through a Gi-coupled cell surface receptor. Potential involvement in angiogenesis. J. Biol. Chem. 174:35343-35350.

16. Lee, M.J., et al. 1998. Sphingosine 1-phosphate as a ligand for the G-protein-coupled receptor Edg-1. Science. 179:1552-1559.

17. Carmelett, P. 2000. Mechanisms of angiogenesis and arteriogenesis. Nature Med. 6:389-395.

18. Liu, Y., et al. 2000. Edg-1, the G protein-coupled receptor for sphingosine 1-phosphate, is essential for vascular maturation. J. Clin. Invest. 106:951-961.

19. Thurston, G., et al. 2000. Leakage-resistant blood vessels in mice transgenically over-expressing angiopoietin-1. Science. 286:2511-2514.

20. Goetzl, E.J., and Songzhu, A. 1998. Diversity of cellular receptors and functions for the lysophospholipid growth factors lysophosphatidic acid and sphingosine 1-phosphate. FASEB J. 12:1589-1598.

21. Hisano, N., et al. 1999. Induction and suppression of apoptosis by sphingolipids: a possible in vitro model for cell-cell interactions between platelets and endothelial cells. Blood. 93:4293-4299.

22. An, S., Zheng, Y., and Bleu, T. 2000. Sphingosine 1-phosphate-induced cell proliferation, survival and related signaling events mediated by $\mathrm{G}$ protein-coupled receptors Edg-3 and Edg-5. J. Biol. Chem. 275:288-296.

23. Hla, T., and Maciag, T. 1990. An abundant transcript induced in differentiating human endothelial cells encodes a poly peptide with structural similarities to G-protein-coupled receptors. J. Biol. Chem. 165:9308-9313.

24. Zondag, G.C.M., Postma, F.R., van Etten, I., Verlaan, I., and Moolenaar, W.H. 1998. Sphingosine-1-phosphate signaling through the G-proteincoupled receptor Edg-1. Biochem. J. 330:605-609.

25. An, S., Dickens, M.A., Bleu, T., Hallmark, O.G., and Goetzl, E.J. 1997. Molecular cloning of the Edg-2 protein and its identification as a functional cellular receptor for lysophosphatidic acid. Biochem. Biophys. Res. Commun. 231:619-622.

26. Pyne, S., and Pyne, N.J. 2000. Sphingosine 1-phosphate signaling in mammalian cells. Biochem. J. 349:385-402.

27. Liu, F., et al. 2001. Differential regulation of sphingosine 1-phosphateand VEGF-induced endothelial cell chemotaxis: Involvement of $\mathrm{G}_{\mathrm{i \alpha 2}-}$ linked Rho kinase activity. Am. J. Res. Cell. Molec. Biol. 24:711-719.

28. Garcia, J.G.N., Painter, R.G., Fenton, J.W., English, D., and Callahan, K.S. 1990. Thrombin-induced prostacyclin biosynthesis in human endothelium: role of guanine nucleotide regulatory proteins in stimulus/coupling responses. J. Cell. Physiol. 142:186-193.

29. Giaever, I., and Keese, C.R. 1993. A morphological biosensor for mammalian cells. Nature. 366:591-592.

30. Garcia, J.G.N., et al. 1997. Mechanisms of ionomycin-induced endothelial cell barrier dysfunction. Am. J. Physiol. 273:L172-L184.

31. Tiruppathi, C., Malik, A.B., Del Vecchio, P.J., Keese, C.R., and Giaever, I. 1992. Electrical method for detection of endothelial cell shape change in real time: assessment of endothelial barrier function. Proc. Natl. Acad. Sci. USA. 89:7919-7923.

32. Garcia, J.G.N., et al. 2000. Diperoxovanadate alters endothelial cell focal contacts and increases permeability: Role of tyrosine phosphorylation. J. Appl. Physiol. 89:2333-2343.

33. Gilchrist, A., et al. 1998. Antagonists of the receptor-G protein interface block $\mathrm{G}_{\mathrm{i}}$-coupled signal transduction. J. Biol. Chem. 273:14912-14919.

34. Lee, O.H., et al. 1999. Sphingosine 1-phosphate induces angiogenesis: its angiogenic action and signaling mechanism in human umbilical vein endothelial cells. Biochem. Biophys. Res. Comm. 264:743-750.

35. Lee, O.H., et al. 2000. Sphingosine 1-phosphate stimulates tyrosine phosphorylation of focal adhesion kinases and chemotactic motility of endothelial cells via the $G_{i}$ protein-linked phospholipase $C$ pathway. Biochem. Biophys. Res. Comm. 268:47-53.

36. Wang, F., et al. 1999. Sphingosine-1-phosphate induces angiogenesis: its angiogenic action and signaling mechanism in human umbilical vein endothelial cells. Biochem. Biophys. Res. Comm. 264:743-750.

37. Garcia, J.G.N., et al. 1993. Thrombin receptor activating peptides induce $\mathrm{Ca}^{2+}$ mobilization, barrier dysfunction, prostaglandin synthesis and platelet-derived growth factor mRNA expression in cultured endothelium. J. Cell. Physiol. 156:541-549.

38. Verin, A.D., et al. 2000. Role of Ras-dependent ERK activation in phorbol ester-induced endothelial cell barrier dysfunction. Am. J. Physiol. 
Lung Cell. Mol. Physiol. 279:L360-L370.

39. Garcia, J.G.N., and Schaphorst, K.L. 1995. Regulation of endothelial cell gap formation and paracellular permeability. J. Invest. Med. 43:117-126.

40. Stevens, T., Garcia, J.G.N., Shasby, D.M., Bhattacharya, J., and Malik, A.B. 2000. EB2000 symposium report: mechanisms regulating endothelial cell barrier function. Am J. Physiol. Lung Cell. Mol. Physiol. 279:L419-L422.

41. Garcia, J.G.N., Davis, H.W., and Patterson, C.E. 1995. Regulation of endothelial cell gap formation and barrier dysfunction. Role of myosin light chain phosphorylation. J. Cell. Physiol. 163:510-522.

42. Garcia, J.G.N., Lazar, V., Gilbert-McClain, L., Gallagher, P.J., and Verin, A. 1997. Myosin light chain kinase in endothelium: molecular cloning and regulation. Am. J. Respir. Cell Mol. Biol. 16:489-494.

43. Edwards, D.C., Sanders, L.C., Bokoch, G.M., and Gill, G.N. 1999. Activation of LIM-kinase by PAK-1 couples Rac/Cdc43 GTPase signaling to actin cytoskeletal dynamics. Nat. Cell. Biol. 1:253-259.

44. Yang, N., et al. 1998. Cofilin phosphorylation by LIM-kinase 1 and its role in Rac-mediated actin reorganization. Nature. 393:809-812.

45. Natarajan, V., Jayaram, H.N., Scribner, W.M., and Garcia, J.G.N. 1994. Activation of endothelial cell phospholipase D by sphingosine and sphingosine-1-phosphate. Am. J. Respir. Cell Mol. Biol. 11: 221-229.

46. Spiegel, S., and Milstien, S. 2000. Sphingosine 1-phosphate: signaling inside and out. FEBS Lett. 476:55-57.

47. Patterson, C.E., Davis, H.W., Schaphorst, K., and Garcia, J.G.N. 1995. Mechanisms of pertussis toxin-induced barrier dysfunction in bovine pulmonary artery endothelial cell monolayers. Am J. Physiol. Lung Cell. Mol. Physiol. 268:L926-L934.

48. Ohomori, T., et al. 2001. Gi-mediated Cas tyrosine phosphorylation in vascular endothelial cells stimulated with sphingosine 1-phosphate: possible involvement in cell motility enhancement in cooperation with Rho-mediated pathways. J. Biol. Chem. 276:5274-5280.

49. Kozawa, O., et al. 1999. Sphingosine 1-phosphate regulates heat shock protein 27 induction by a 38 MAP kinase-dependent mechanism in aortic smooth muscle cells. Exp. Cell. Res. 250:376-380.

50. Miura, Y., et al. 2000. Rho-mediated phosphorylation of focal adhesion kinase and myosin light chain in human endothelial cells stimulated with sphingosine 1-phosphate, a bioactive lysophospholipid released from activated platelets. J. Biochem. 127:909-914.

51. Amano, M. et al. 1996. Phosphorylation and activation of myosin by Rho-associated kinase (Rho-kinase). J. Biol. Chem. 271:20246-20249.

52. Chew, T.L., Masaracchia, R.A., Goeckeler, Z.M., and Wysolmerski, R.B. 1998. Phosphorylation and activation of myosin light chain by $\mathrm{p} 21-$ activated kinase ( $\gamma$-PAK). J. Muscle Res. Cell Motil. 19:839-854.

53. Knaus, U.G., Morris, S., Dong, H.J., Chernoff, J., and Bokoch, G.M. 1995. Regulation of human leukocyte $\mathrm{p} 21$-activated kinases through G-protein-coupled receptors. Science. 269:221-223.

54. Bokoch, G.M., et al. 1998. A GTPase-independent mechanism of p21activated kinase activation. J. Biol. Chem. 14:8137-8144.

55. Sanders, L.C., Matsumura, F., Bokoch, G.M.N., and de Lanerolle, P. 1999. Inhibition of myosin light chain kinase by $\mathrm{p} 21$-activated kinase. Science. 283:2083-2085.

56. Birukov, K.G., et al. 2001. Differential regulation by tyrosine phosphorylation of alternatively spliced endothelial cell MLCK isoforms. J. Biol. Chem. 276:8567-8573.

57. Sells, M.A., Pfaff, A., and Chernoff, J. 2000. Temporal and spatial distribution of activated Pak1 in fibroblasts. J. Cell. Biol. 151:1449-1458.

58. Aizawa, H., et al. 1999. Hyperosmotic stress-induced reorganization of actin bundles in Dictyostelium cells over-expressing cofilin. Genes Cells. 4:311-324.

59. Stanyon, C.A., and Bernard, O. LIM-kinase 1. 1999. Int. J. Biochem Cell Biol. 31:389-394.

60. Moon, A.L., Janmey, P.A., Louie, K.A., and Drubin, D.G. 1993. Cofilin is an essential component of the yeast cortical cytoskeleton. J. Cell Biol. 120:421-435.

61. Arber, S., et al. 1998. Regulation of actin dynamics through phosphorylation of cofilin by LIM-kinase. Nature. 393:805-809.

62. Ohashi, K., et al. 2000. Rho-associated kinase ROCK activates LIMkinase 1 by phosphorylation at threonine 508 within the activation loop. J. Biol. Chem. 275:3577-3582.

63. Meberg, P.J., and Bamburg, J.R. 2000. Increase in neurite outgrowth mediated by over-expression of actin depolymerizing factor. J. Neurosi. 20:2459-2469. 